Mathematical Modelling and Analysis

Volume 22 Number 2, March 2017, 121-139

https://doi.org/10.3846/13926292.2017.1274920

(c) Vilnius Gediminas Technical University, 2017
Publisher: Taylor\&Francis and VGTU

http://www.tandfonline.com/TMMA

ISSN: $1392-6292$

eISSN: $1648-3510$

\title{
A Study of Nonlinear Fractional-Order Boundary Value Problem with Nonlocal Erdélyi-Kober and Generalized Riemann-Liouville Type Integral Boundary Conditions
}

\section{Bashir Ahmad $^{a}$, Sotiris K. Ntouyas ${ }^{b, a}$, Jessada Tariboon ${ }^{c}$ and Ahmed Alsaedi ${ }^{a}$}

${ }^{a}$ Nonlinear Analysis and Applied Mathematics (NAAM)-Research Group Department of Mathematics, Faculty of Science, P.O. Box 80203, Jeddah 21589, Saudi Arabia

${ }^{b}$ University of Ioannina

Department of Mathematics, 45110 Ioannina, Greece

${ }^{c}$ Nonlinear Dynamic Analysis Research Center

Department of Mathematics, Faculty of Applied Science, King Mongkut's

University of Technology North Bangkok, Bangkok, 10800 Thailand

E-mail(corresp.): bashirahmad_qau@yahoo.com

E-mail: aalsaedi@hotmail.com

E-mail: sntouyas@uoi.gr

E-mail: jessada.t@sci.kmutnb.ac.th

Received April 7, 2016; revised December 14, 2016; published online March 1, 2017

\begin{abstract}
We investigate a new kind of nonlocal boundary value problems of nonlinear Caputo fractional differential equations supplemented with integral boundary conditions involving Erdélyi-Kober and generalized Riemann-Liouville fractional integrals. Existence and uniqueness results for the given problem are obtained by means of standard fixed point theorems. Examples illustrating the main results are also discussed.
\end{abstract}

Keywords: Caputo fractional derivative, Erdélyi-Kober, generalized Riemann-Liouville fractional integral, fractional integral, fixed point.

AMS Subject Classification: 34A08; 34B10.

\section{Introduction}

Hemodynamic conditions (blood velocity and pressure as a function of space and time) can be completely characterized by computational fluid dynamics (CFD) techniques under appropriate boundary data. The idea of assuming the 
geometry of blood vessel to be of circular type upstream the inlet is not always justifiable. An alternative and accurate approach to model the blood flow problems is to consider integral boundary conditions, for details, see [2] and references cited therein. Integral boundary conditions also help to regularize ill-posed parabolic backward problems in time partial differential equations [33].

The recent surge in developing theoretical and numerical aspects of fractional calculus is mainly due to widespread applications of fractional-order operators in mathematical modeling of several real world phenomena. Examples include acoustics, viscoelasticity, signal and image processing, chemical processes, control theory, biomathematics, biomedical, etc. For more details and explanations, see the texts $[16,18,23,26,27]$. We emphasize that fractionalorder differential and integral operators help to gather useful information about hereditary and memory characteristics of the processes and materials involved in the phenomena.

Fractional differential equations supplemented with a variety of initial and boundary conditions have been investigated by several researchers and the literature on the topic is now much enriched. For examples and recent development of the topic, see $[1,3,4,5,6,7,9,12,13,21,22,24,25,30,31,32]$ and the references cited therein.

Classical, Riemann-Liouville or Hadamard or Erdélyi-Kober type integrals appear in the study of fractional-order boundary value problems with integral boundary conditions. In [15], the author expressed Riemann-Liouville and Hadamard fractional integrals into a single form, which is known as generalized Riemann-Liouville fractional integral (see Definition 2).

In this paper, we introduce a new class of boundary value problems of Caputo fractional differential equations supplemented with Erdélyi-Kober and generalized Riemann-Liouville fractional integral boundary conditions. In precise terms, we consider the following boundary value problem

$$
\left\{\begin{array}{l}
{ }^{c} D^{q} x(t)=f(t, x(t)), \quad t \in[0, T], \\
x(0)=\alpha \frac{\eta \xi^{-\eta(\delta+\gamma)}}{\Gamma(\delta)} \int_{0}^{\zeta} \frac{s^{\eta \gamma+\eta-1} x(s)}{\left(\zeta^{\eta}-s^{\eta}\right)^{1-\delta}} d s:=\alpha I_{\eta}^{\gamma, \delta} x(\zeta), \\
x(T)=\beta \frac{\rho^{1-p}}{\Gamma(p)} \int_{0}^{\xi} \frac{s^{\rho-1} x(s)}{\left(\xi^{\rho}-s^{\rho}\right)^{1-p}} d s:=\beta^{\rho} I^{p} x(\xi), \quad 0<\zeta, \xi<T,
\end{array}\right.
$$

where ${ }^{c} D^{q}$ is the Caputo fractional derivative of order $1<q \leq 2, f:[0, T] \times \mathbb{R} \rightarrow$ $\mathbb{R}$ is a continuous function, $I_{\eta}^{\gamma, \delta}$ is Erdélyi-Kober fractional integrals of order $\delta>0, \eta>0, \gamma \in \mathbb{R},{ }^{\rho} I^{p}$ is the generalized Riemann-Liouville fractional integral of order $p>0, \rho>0$ and $\alpha, \beta \in \mathbb{R}$.

We remark that Erdélyi-Kober fractional integral operator, introduced by Arthur Erdélyi and Hermann Kober [11] in 1940, is useful in solving single, dual and triple integral equations possessing special functions of mathematical physics in their kernels. For details and applications of the Erdélyi-Kober fractional integrals, for instance, see [10,17, 19, 29,34].

The paper is organized as follows: In Section 2 we will present some useful preliminaries and lemmas. Section 3 deals with the existence and uniqueness 
results for the boundary value problem (1.1) which are established via Krasnoselskii fixed point theorem, Laray-Schauder nonlinear alternative, contraction mapping principle, nonlinear contractions, Schaefer fixed point theorem, and Laray-Schauder degree theory. The paper concludes with illustrative examples.

\section{Preliminaries}

This section is devoted to some preliminary concepts of fractional calculus that we need in the forthcoming analysis [16].

Definition 1. The fractional integral of order $q$ with the lower limit zero for a function $\mathrm{f}$ is defined as

$$
J^{q} f(t)=\frac{1}{\Gamma(q)} \int_{0}^{t} \frac{f(s)}{(t-s)^{1-q}} d s, \quad t>0, \quad q>0,
$$

provided the right hand-side is point-wise defined on $[0, \infty)$, where $\Gamma(\cdot)$ is the gamma function, which is defined by $\Gamma(q)=\int_{0}^{\infty} t^{q-1} e^{-t} d t$.

Definition 2. [15] The generalized fractional integral of order $q>0$ and $\rho>0$ of a function $f(t)$, for all $0<t<\infty$, is defined as

$$
{ }^{\rho} I^{q} f(t)=\frac{\rho^{1-q}}{\Gamma(q)} \int_{0}^{t} \frac{s^{\rho-1} f(s)}{\left(t^{\rho}-s^{\rho}\right)^{1-q}} d s,
$$

provided the right-hand side is point-wise defined on $(0, \infty)$.

Remark 1. From the above definition it follows that $\rho=1$ corresponds to the standard Riemann-Liouville fractional integral, while $\rho \rightarrow 0$ yields the Hadamard fractional integral

$$
\lim _{\rho \rightarrow 0}{ }^{\rho} I^{q} f(t)=\frac{1}{\Gamma(q)} \int_{0}^{t}\left(\ln \frac{t}{s}\right)^{q-1} \frac{f(s)}{s} d s .
$$

For further details, see [15].

Definition 3. The Erdélyi-Kober fractional integral of order $\delta>0$ with $\eta>0$ and $\gamma \in \mathbb{R}$ of a continuous function $f:(0, \infty) \rightarrow \mathbb{R}$ is defined by

$$
I_{\eta}^{\gamma, \delta} f(t)=\frac{\eta t^{-\eta(\delta+\gamma)}}{\Gamma(\delta)} \int_{0}^{t} \frac{s^{\eta \gamma+\eta-1} f(s)}{\left(t^{\eta}-s^{\eta}\right)^{1-\delta}} d s,
$$

provided the right side is pointwise defined on $\mathbb{R}_{+}$.

Remark 2. For $\eta=1$ the above operator is reduced to the Kober operator

$$
I_{1}^{\gamma, \delta} f(t)=\frac{t^{-(\delta+\gamma)}}{\Gamma(\delta)} \int_{0}^{t} \frac{s^{\gamma} f(s)}{(t-s)^{1-\delta}} d s, \quad \gamma, \delta>0,
$$

that was introduced for the first time by Kober in [19]. For $\gamma=0$, the Kober operator is reduced to the Riemann-Liouville fractional integral with a power weight:

$$
I_{1}^{0, \delta} f(t)=\frac{t^{-\delta}}{\Gamma(\delta)} \int_{0}^{t} \frac{f(s)}{(t-s)^{1-\delta}} d s, \quad \delta>0 .
$$


Definition 4. The Riemann-Liouville fractional derivative of order $q>0, n-$ $1<q<n, n \in N$, is defined as

$$
D_{0+}^{q} f(t)=\frac{1}{\Gamma(n-q)}\left(\frac{d}{d t}\right)^{n} \int_{0}^{t}(t-s)^{n-q-1} f(s) d s
$$

where the function $f(t)$ has absolutely continuous derivative up to order $(n-1)$.

Definition 5. The Caputo derivative of order $q$ for a function $f:[0, \infty) \rightarrow \mathbb{R}$ can be written as

$$
{ }^{c} D^{q} f(t)=D_{0+}^{q}\left(f(t)-\sum_{k=0}^{n-1} \frac{t^{k}}{k !} f^{(k)}(0)\right), \quad t>0, \quad n-1<q<n .
$$

Remark 3. If $f(t) \in C^{n}[0, \infty)$, then

$$
{ }^{c} D^{q} f(t)=\frac{1}{\Gamma(n-q)} \int_{0}^{t} \frac{f^{(n)}(s)}{(t-s)^{q+1-n}} d s=I^{n-q} f^{(n)}(t), t>0, n-1<q<n .
$$

Lemma 1. Let $\delta, \eta>0$ and $\gamma, q \in \mathbb{R}$. Then we have

$$
I_{\eta}^{\gamma, \delta} t^{q}=\frac{t^{q} \Gamma(\gamma+(q / \eta)+1)}{\Gamma(\gamma+(q / \eta)+\delta+1)} .
$$

Proof. Recall the definition of beta function and its property:

$$
B(x, y)=\int_{0}^{1} u^{x-1}(1-u)^{y-1} d u \quad \text { and } \quad B(x, y)=\frac{\Gamma(x) \Gamma(y)}{\Gamma(x+y)},
$$

for $x, y>0$. From Definition 3, we have

$$
\begin{aligned}
I_{\eta}^{\gamma, \delta} t^{q} & =\frac{\eta t^{-\eta(\delta+\gamma)}}{\Gamma(\delta)} \int_{0}^{t} \frac{s^{\eta \gamma+\eta-1} \cdot s^{q}}{\left(t^{\eta}-s^{\eta}\right)^{1-\delta}} d s=\frac{t^{q}}{\Gamma(\delta)} \int_{0}^{1} u^{\gamma+\frac{q}{\eta}}(1-u)^{\delta-1} d u \\
& =\frac{t^{q}}{\Gamma(\delta)} B\left(\gamma+\frac{q}{\eta}+1, \delta\right)=\frac{t^{q} \Gamma(\gamma+(q / \eta)+1)}{\Gamma(\gamma+(q / \eta)+\delta+1)} .
\end{aligned}
$$

The proof is complete.

Lemma 2. Let constants $q>0$ and $p>0$. Then:

$$
{ }^{\rho} I^{q} t^{p}=\frac{\Gamma(p+\rho / \rho)}{\Gamma((p+\rho q+\rho) / \rho)} \frac{t^{p+\rho q}}{\rho^{q}} .
$$

Proof. From Definition 2, we have

$$
\begin{aligned}
{ }^{\rho} I^{q} t^{p} & =\frac{\rho^{1-q}}{\Gamma(q)} \int_{0}^{t} \frac{s^{\rho-1} s^{p}}{\left(t^{\rho}-s^{\rho}\right)^{1-q}} d s=\frac{\rho^{1-q}}{\Gamma(q)} \frac{t^{p+\rho q}}{\rho} \int_{0}^{1} \frac{u^{\frac{p}{\rho}}}{(1-u)^{1-q}} d u \\
& =\frac{\rho^{1-q}}{\Gamma(q)} \frac{t^{p+\rho q}}{\rho} B\left(\frac{p+\rho}{\rho}, q\right)=\frac{t^{p+\rho q}}{\rho^{q}} \frac{\Gamma\left(\frac{p+\rho}{\rho}\right)}{\Gamma\left(\frac{p+\rho q+\rho}{\rho}\right)} .
\end{aligned}
$$

This completes the proof. 
Lemma 3. For any $y \in C[0, T]$, a function $x \in C^{2}[0, T]$ is a solution of the linear fractional boundary value problem

$$
\left\{\begin{array}{l}
{ }^{c} D^{q} x(t)=y(t), \quad 1<q \leq 2, \\
x(0)=\alpha I_{\eta}^{\gamma, \delta} x(\zeta), \quad x(T)=\beta^{\rho} I^{p} x(\xi), \quad 0<\zeta, \xi<T,
\end{array}\right.
$$

if and only if

$$
\begin{aligned}
x(t)= & J^{q} y(t)+\frac{\alpha}{\Lambda}\left(v_{4}-t v_{3}\right) I_{\eta}^{\gamma, \delta} J^{q} y(\zeta) \\
& +\frac{1}{\Lambda}\left(v_{2}+t v_{1}\right)\left(\beta^{\rho} I^{p} J^{q} y(\xi)-J^{q} y(T)\right),
\end{aligned}
$$

where

$$
\begin{array}{ll}
v_{1}=1-\alpha \frac{\Gamma(\gamma+1)}{\Gamma(\gamma+\delta+1)}, & v_{2}=\alpha \zeta \frac{\Gamma(\gamma+1 / \eta+1)}{\Gamma(\gamma+1 / \eta+\delta+1)}, \\
v_{3}=1-\beta \frac{\xi^{\rho p}}{\rho^{\beta}} \frac{1}{\Gamma(p+1)}, & v_{4}=T-\beta \frac{\xi^{\rho p+1}}{\rho^{p}} \frac{\Gamma((1+\rho) / \rho)}{\Gamma((1+\rho p+\rho) / \rho)}
\end{array}
$$

and $\Lambda=v_{1} v_{4}+v_{2} v_{3} \neq 0$.

Proof. It is well known [16] that the general solution of fractional differential equation in (2.1) can be written as

$$
x(t)=c_{0}+c_{1} t+J^{q} y(t)
$$

where $c_{0}, c_{1} \in \mathbb{R}$ are arbitrary constants.

Applying the Erdélyi-Kober fractional integral operator of order $\delta>0$ with $\eta>0$ and $\gamma \in \mathbb{R}$ on (2.4) and using Lemma 1, we obtain

$$
I_{\eta}^{\gamma, \delta} x(t)=I_{\eta}^{\gamma, \delta} J^{q} y(t)+c_{1} t \frac{\Gamma(\gamma+1 / \eta+1)}{\Gamma(\gamma+1 / \eta+\delta+1)}+c_{0} \frac{\Gamma(\gamma+1)}{\Gamma(\gamma+\delta+1)} .
$$

Using (2.5) in the first condition of (2.1), we get

$$
c_{0}=\alpha I_{\eta}^{\gamma, \delta} J^{q} y(\zeta)+\alpha c_{0} \frac{\Gamma(\gamma+1)}{\Gamma(\gamma+\delta+1)}+\alpha c_{1} \zeta \frac{\Gamma(\gamma+1 / \eta+1)}{\Gamma(\gamma+1 / \eta+\delta+1)} .
$$

Applying the generalized fractional integral operator on (2.4) and using Lemma 2 , we obtain

$$
{ }^{\rho} I^{p} x(t)={ }^{\rho} I^{p} J^{q} y(t)+c_{0} \frac{t^{\rho p}}{\rho^{p}} \frac{1}{\Gamma(p+1)}+c_{1} \frac{t^{\rho p+1}}{\rho^{p}} \frac{\Gamma((1+\rho) / \rho)}{\Gamma((1+\rho p+\rho) / \rho)} .
$$

Using (2.7) in the second boundary condition of (2.1), we get

$$
J^{q} y(T)+c_{0}+c_{1} T=\beta^{\rho} I^{p} J^{q} y(\xi)+c_{0} \beta \frac{\xi^{\rho p}}{\rho^{p}} \frac{1}{\Gamma(p+1)}+c_{1} \beta \frac{\xi^{\rho p+1}}{\rho^{p}} \frac{\Gamma\left(\frac{1+\rho}{\rho}\right)}{\Gamma\left(\frac{1+\rho p+\rho}{\rho}\right)} .
$$


Using the notations (2.3) in (2.6) and (2.8), we get the system

$$
\begin{aligned}
& v_{1} c_{0}-v_{2} c_{1}=\alpha I_{\eta}^{\gamma, \delta} J^{q} y(\zeta), \\
& v_{3} c_{0}+v_{4} c_{1}=\beta^{\rho} I^{p} J^{q} y(\xi)-J^{q} y(T),
\end{aligned}
$$

which, on solving for $c_{0}$ and $c_{1}$, yields

$$
\begin{aligned}
& c_{0}=\frac{1}{\Lambda}\left\{\alpha v_{4} I_{\eta}^{\gamma, \delta} J^{q} y(\zeta)+v_{2}\left(\beta^{\rho} I^{p} J^{q} y(\xi)-J^{q} y(T)\right)\right\}, \\
& c_{1}=\frac{1}{\Lambda}\left\{v_{1}\left(\beta^{\rho} I^{p} J^{q} y(\xi)-J^{q} y(T)\right)-\alpha v_{3} I_{\eta}^{\gamma, \delta} J^{q} y(\zeta)\right\} .
\end{aligned}
$$

Substituting the values of $c_{0}, c_{1}$ in (2.4), we get (2.2). Conversely, it follows by direct computation that the integral equation (2.2) satisfies the problem (2.1). This completes the proof.

\section{$3 \quad$ Existence results}

We denote by $\mathcal{C}=C([0, T], \mathbb{R})$ the Banach space of all continuous functions from $[0, T] \rightarrow \mathbb{R}$ endowed with a topology of uniform convergence with the norm defined by $\|x\|=\sup \{|x(t)|: t \in[0, T]\}$. Also by $L^{1}([0, T], \mathbb{R})$ we denote the Banach space of measurable functions $x:[0, T] \rightarrow \mathbb{R}$ which are Lebesgue integrable and normed by $\|x\|_{L^{1}}=\int_{0}^{T}|x(t)| d t$.

In view of Lemma 3 , we define an operator $\mathcal{P}: \mathcal{C} \rightarrow \mathcal{C}$ by

$$
\begin{aligned}
& (\mathcal{P} x)(t)=J^{q} f(s, x(s))(t)+\frac{\alpha}{\Lambda}\left(v_{4}-t v_{3}\right) I_{\eta}^{\gamma, \delta} J^{q} f(s, x(s))(\zeta) \\
& +\frac{1}{\Lambda}\left(v_{2}+t v_{1}\right)\left(\beta^{\rho} I^{p} J^{q} f(s, x(s))(\xi)-J^{q} f(s, x(s))(T)\right), t \in[0, T] .
\end{aligned}
$$

In the sequel, we use the following expressions:

$$
{ }^{\rho} I^{p} f(s, x(s))(y)=\frac{\rho^{1-p}}{\Gamma(p)} \int_{0}^{y} \frac{s^{\rho-1} f(s, x(s))}{\left(y^{\rho}-s^{\rho}\right)^{1-p}} d s,
$$

for $y \in[0, T]$ and

$$
I_{\eta}^{\gamma, \delta} J^{q} f(s, x(s))(\xi)=\frac{\eta \xi^{-\eta(\delta+\gamma)}}{\Gamma(q) \Gamma(\delta)} \int_{0}^{\xi} \int_{0}^{r} \frac{r^{\eta \gamma+\eta-1}(r-s)^{q-1}}{\left(\xi^{\eta}-r^{\eta}\right)^{1-\delta}} f(s, x(s)) d s d r
$$

where $\xi \in(0, T)$.

For convenience, we set a constant

$$
\begin{aligned}
\Psi: & =\frac{T^{q}}{\Gamma(q+1)}+\frac{|\alpha|\left(\left|v_{4}\right|+T\left|v_{3}\right|\right)}{|\Lambda|} \frac{\zeta^{q} \Gamma(\gamma+(q / \eta)+1)}{\Gamma(q+1) \Gamma(\gamma+(q / \eta)+\delta+1)} \\
& +\frac{\left(\left|v_{2}\right|+T\left|v_{1}\right|\right)}{|\Lambda|}\left(\frac{1}{\Gamma(q+1)} \frac{\xi^{q+\rho p}}{\rho^{p}} \frac{\Gamma\left(\frac{q+\rho}{\rho}\right)}{\Gamma\left(\frac{q+\rho p+\rho}{\rho}\right)}+\frac{T^{q}}{\Gamma(q+1)}\right) .
\end{aligned}
$$

In the following subsections we present our main results for problem (1.1) by making use of a variety of fixed point theorems. 


\subsection{Existence result via Krasnoselskii's fixed point theorem}

Lemma 4. (Krasnoselskii's fixed point theorem) [20]. Let $M$ be a closed, bounded, convex and nonempty subset of a Banach space $X$. Let $A, B$ be the operators such that $(a) A x+B y \in M$ whenever $x, y \in M ;(b) A$ is compact and continuous; $(c) B$ is a contraction mapping. Then there exists $z \in M$ such that $z=A z+B z$.

Theorem 1. Let $f:[0, T] \times \mathbb{R} \rightarrow \mathbb{R}$ be a continuous function satisfying the assumptions:

$\left(H_{1}\right)$ there exists a positive constant $L$ such that $|f(t, x)-f(t, y)| \leq L|x-y|$, for each $t \in[0, T]$ and $x, y \in \mathbb{R}$.

$\left(H_{2}\right)|f(t, x)| \leq \varphi(t), \quad \forall(t, x) \in[0, T] \times \mathbb{R}$ and $\varphi \in C\left([0, T], \mathbb{R}^{+}\right)$.

Then problem (1.1) has at least one solution on $[0, T]$ provided that

$$
\begin{aligned}
\Psi_{1} & :=L\left\{\frac{|\alpha|\left(\left|v_{4}\right|+T\left|v_{3}\right|\right)}{|\Lambda|} \frac{\zeta^{q} \Gamma(\gamma+(q / \eta)+1)}{\Gamma(q+1) \Gamma(\gamma+(q / \eta)+\delta+1)}\right. \\
& \left.+\frac{\left(\left|v_{2}\right|+T\left|v_{1}\right|\right)}{|\Lambda|}\left(\frac{1}{\Gamma(q+1)} \frac{\xi^{q+\rho p}}{\rho^{p}} \frac{\Gamma((q+\rho) / \rho)}{\Gamma((q+\rho p+\rho) / \rho)}+\frac{T^{q}}{\Gamma(q+1)}\right)\right\}<1 .
\end{aligned}
$$

Proof. We define operator $\mathcal{P}_{1}, \mathcal{P}_{2}: \mathcal{C} \rightarrow \mathcal{C}$ by

$$
\begin{aligned}
\mathcal{P}_{1} x(t)= & J^{q} f(s, x(s))(t), \quad t \in[0, T] \\
\mathcal{P}_{2} y(t)= & \frac{\alpha}{\Lambda}\left(v_{4}-t v_{3}\right) I_{\eta}^{\gamma, \delta} J^{q} f(s, y(s))(\zeta) \\
& +\frac{1}{\Lambda}\left(v_{2}+t v_{1}\right)\left(\beta^{\rho} I^{p} J^{q} f(s, y(s))(\xi)-J^{q} f(s, y(s))(T)\right), \quad t \in[0, T] .
\end{aligned}
$$

Setting $\sup _{t \in[0, T]} \varphi(t)=\|\varphi\|$ and choosing $\rho \geq\|\varphi\| \Psi$, where $\Psi$ is defined by (3.2), we consider $B_{\rho}=\{x \in \mathcal{C}:\|x\| \leq \rho\}$. For any $x, y \in B_{\rho}$, we have

$$
\begin{aligned}
& \left|\mathcal{P}_{1} x(t)+\mathcal{P}_{2} y(t)\right| \leq \sup _{t \in[0, T]}\left\{J^{q}|f(s, x(s))|(t)+\frac{|\alpha|}{|\Lambda|}\left(\left|v_{4}\right|+T\left|v_{3}\right|\right)\right. \\
& \quad \times I_{\eta}^{\gamma, \delta} J^{q}|f(s, y(s))|(\zeta)+\frac{1}{|\Lambda|}\left(\left|v_{2}\right|+T\left|v_{1}\right|\right) \\
& \left.\quad \times\left(|\beta|^{\rho} I^{p} J^{q}|f(s, y(s))|(\xi)+J^{q}|f(s, y(s))|(T)\right)\right\} \\
& \leq\|\varphi\|\left\{\frac{T^{q}}{\Gamma(q+1)}+\frac{|\alpha|\left(\left|v_{4}\right|+T\left|v_{3}\right|\right)}{|\Lambda|} \frac{\zeta^{q} \Gamma(\gamma+(q / \eta)+1)}{\Gamma(q+1) \Gamma(\gamma+(q / \eta)+\delta+1)}\right. \\
& \left.\quad+\frac{\left(\left|v_{2}\right|+T\left|v_{1}\right|\right)}{|\Lambda|}\left(\frac{1}{\Gamma(q+1)} \frac{\xi^{q+\rho p}}{\rho^{p}} \frac{\Gamma\left(\frac{q+\rho}{\rho}\right)}{\Gamma\left(\frac{q+\rho p+\rho}{\rho}\right)}+\frac{T^{q}}{\Gamma(q+1)}\right)\right\}=\|\varphi\| \Psi \leq \rho .
\end{aligned}
$$

This shows that $\mathcal{P}_{1} x+\mathcal{P}_{2} y \in B_{\rho}$. Using the assumption $\left(H_{1}\right)$, we get

$$
\left\|\mathcal{P}_{2} x-\mathcal{P}_{2} y\right\| \leq L\left\{\frac{|\alpha|\left(\left|v_{4}\right|+T\left|v_{3}\right|\right)}{|\Lambda|} \frac{\zeta^{q} \Gamma(\gamma+(q / \eta)+1)}{\Gamma(q+1) \Gamma(\gamma+(q / \eta)+\delta+1)}\right.
$$




$$
\left.+\frac{\left(\left|v_{2}\right|+T\left|v_{1}\right|\right)}{|\Lambda|}\left(\frac{1}{\Gamma(q+1)} \frac{\xi^{q+\rho p}}{\rho^{p}} \frac{\Gamma\left(\frac{q+\rho}{\rho}\right)}{\Gamma\left(\frac{q+\rho p+\rho}{\rho}\right)}+\frac{T^{q}}{\Gamma(q+1)}\right)\right\}\|x-y\|,
$$

which, in view of (3.3), implies that $\mathcal{P}_{2}$ is a contraction.

Continuity of $f$ implies that the operator $\mathcal{P}_{1}$ is continuous. Also, $\mathcal{P}_{1}$ is uniformly bounded on $B_{\rho}$ as

$$
\left\|\mathcal{P}_{1} x\right\| \leq \frac{T^{q}}{\Gamma(q+1)}\|\varphi\| .
$$

Next we prove the compactness of the operator $\mathcal{P}_{1}$. Let $\sup _{(t, x) \in[0, T] \times B_{\rho}}|f(t, x)|=\bar{f}<\infty$. Then, for $t_{1}, t_{2} \in[0, T]$ with $t_{1}<t_{2}$, we obtain

$$
\begin{aligned}
& \left|\mathcal{P}_{1} x\left(t_{2}\right)-\mathcal{P}_{1} x\left(t_{1}\right)\right|=\left|J^{q} f(s, x(s))\left(t_{2}\right)-J^{q} f(s, x(s))\left(t_{1}\right)\right| \\
& \quad \leq \frac{\bar{f}}{\Gamma(q)}\left|\int_{0}^{t_{1}}\left[\left(t_{2}-s\right)^{q-1}-\left(t_{1}-s\right)^{q-1}\right] d s+\int_{t_{1}}^{t_{2}}\left(t_{2}-s\right)^{q-1} d s\right| \\
& \quad \leq \frac{\bar{f}}{\Gamma(q+1)}\left[\left|t_{2}^{q}-t_{1}^{q}\right|+2\left|t_{2}-t_{1}\right|^{q}\right],
\end{aligned}
$$

which tends to zero as $t_{2}-t_{1} \rightarrow 0$ independent of $x$. Thus, $\mathcal{P}_{1}$ is equicontinuous. So $\mathcal{P}_{1}$ is relatively compact on $B_{\rho}$. Hence, by the Arzelá-Ascoli theorem, $\mathcal{P}_{1}$ is compact on $B_{\rho}$. Thus all the assumptions of Lemma 4 are satisfied. Hence the conclusion of Lemma 4 implies that problem (1.1) has at least one solution on $[0, T]$.

\subsection{Existence result via Leray-Schauder's Nonlinear Alternative}

Lemma 5. (Nonlinear alternative for single valued maps) [14]. Let $E$ be a Banach space, $C$ be a closed, convex subset of $E, U$ be an open subset of $C$ and $0 \in U$. Suppose that $\mathcal{A}: \bar{U} \rightarrow C$ is a continuous, compact (that is, $\mathcal{A}(\bar{U})$ is a relatively compact subset of $C$ ) map. Then either

(i) $\mathcal{A}$ has a fixed point in $\bar{U}$, or

(ii) there is a $x \in \partial U$ (the boundary of $U$ in $C$ ) and $\lambda \in(0,1)$ with $x=\lambda \mathcal{A}(x)$.

Theorem 2. Assume that

$\left(H_{3}\right)$ there exists a continuous nondecreasing function $\Phi:[0, \infty) \rightarrow(0, \infty)$ and a function $p \in L^{1}\left([0, T], \mathbb{R}^{+}\right)$such that

$$
|f(t, x)| \leq p(t) \Phi(\|x\|) \text { for each }(t, x) \in[0, T] \times \mathbb{R}
$$

$\left(H_{4}\right)$ there exists a constant $N>0$ such that

$$
N / \Phi(N)>J^{q} p(s)(T)+A_{1}+A_{2},
$$

where

$$
\begin{aligned}
& A_{1}=|\alpha| /|\Lambda|\left(\left|v_{4}\right|+T\left|v_{3}\right|\right) I_{\eta}^{\gamma, \delta} J^{q} p(s)(\zeta) \\
& A_{2}=1 /|\Lambda|\left(\left|v_{2}\right|+T\left|v_{1}\right|\right)\left(|\beta|^{\rho} I^{p} J^{q} p(s)(\xi)+J^{q} p(s)(T)\right) .
\end{aligned}
$$


Then the boundary value problem (1.1) has at least one solution on $[0, T]$.

Proof. We first show that the operator $\mathcal{P}$ (defined by (3.1)) maps bounded sets (balls) into bounded sets in $\mathcal{C}$. For a positive constant $r$, let $B_{r}=\{x \in \mathcal{C}$ : $\|x\| \leq r\}$ be a bounded ball in $\mathcal{C}$. Then for $t \in[0, T]$, we have

$$
\begin{aligned}
|\mathcal{P} x(t)| \leq & J^{q}|f(s, x(s))|(t)+\frac{|\alpha|}{|\Lambda|}\left(\left|v_{4}\right|+T\left|v_{3}\right|\right) I_{\eta}^{\gamma, \delta} J^{q}|f(s, x(s))|(\zeta) \\
& +\frac{1}{|\Lambda|}\left(\left|v_{2}\right|+T\left|v_{1}\right|\right)\left(|\beta|^{\rho} I^{p} J^{q}|f(s, x(s))|(\xi)+J^{q}|f(s, x(s))|(T)\right) \\
\leq & \Phi(\|x\|) J^{q} p(s)(T)+\Phi(\|x\|) \frac{|\alpha|}{|\Lambda|}\left(\left|v_{4}\right|+T\left|v_{3}\right|\right) I_{\eta}^{\gamma, \delta} J^{q} p(s)(\zeta) \\
& +\Phi(\|x\|) \frac{1}{|\Lambda|}\left(\left|v_{2}\right|+T\left|v_{1}\right|\right)\left(|\beta|^{\rho} I^{p} J^{q} p(s)(\xi)+J^{q} p(s)(T)\right)
\end{aligned}
$$

and consequently,

$$
\begin{aligned}
\|\mathcal{P} x\| \leq & \Phi(r)\left\{J^{q} p(s)(T)+\frac{|\alpha|}{|\Lambda|}\left(\left|v_{4}\right|+T\left|v_{3}\right|\right) I_{\eta}^{\gamma, \delta} J^{q} p(s)(\zeta)\right. \\
& \left.+\frac{1}{|\Lambda|}\left(\left|v_{2}\right|+T\left|v_{1}\right|\right)\left(|\beta|^{\rho} I^{p} J^{q} p(s)(\xi)+J^{q} p(s)(T)\right)\right\} .
\end{aligned}
$$

Next we will show that the operator $\mathcal{P}$ maps bounded sets into equicontinuous sets of $\mathcal{C}$. Let $\tau_{1}, \tau_{2} \in[0, T]$ with $\tau_{1}<\tau_{2}$ and $x \in B_{r}$. Then we have

$$
\begin{aligned}
\left|\mathcal{P} x\left(\tau_{2}\right)-\mathcal{P} x\left(\tau_{1}\right)\right| \leq\left|J^{q} f(s, x(s))\left(\tau_{2}\right)-J^{q} f(s, x(s))\left(\tau_{1}\right)\right| \\
\quad+\frac{|\alpha|\left|v_{3}\right|\left|\tau_{2}-\tau_{1}\right|}{|\Lambda|} I_{\eta}^{\gamma, \delta} J^{q}|f(s, x(s))|(T) \\
\quad+\frac{\left|v_{1}\right|\left|\tau_{2}-\tau_{1}\right|}{|\Lambda|}\left(|\beta|^{\rho} I^{p} J^{q}|f(s, x(s))|(\xi)+J^{q}|f(s, x(s))|(T)\right) \\
\leq \frac{\Phi(r)}{\Gamma(q)}\left|\int_{0}^{\tau_{1}}\left[\left(\tau_{2}-s\right)^{q-1}-\left(\tau_{1}-s\right)^{q-1}\right] p(s) d s+\int_{\tau_{1}}^{\tau_{2}}\left(\tau_{2}-s\right)^{q-1} p(s) d s\right| \\
\quad+\frac{\Phi(r)|\alpha|\left|v_{3}\right|\left|\tau_{2}-\tau_{1}\right|}{|\Lambda|} I_{\eta}^{\gamma, \delta} J^{q} p(s)(T) \\
\quad+\frac{\Phi(r)\left|v_{1}\right|\left|\tau_{2}-\tau_{1}\right|}{|\Lambda|}\left(|\beta|^{\rho} I^{p} J^{q} p(s)(\xi)+J^{q} p(s)(T)\right) .
\end{aligned}
$$

As $\tau_{2}-\tau_{1} \rightarrow 0$, the right-hand side of the above inequality tends to zero independently of $x \in B_{r}$. Therefore, by the Arzelá-Ascoli theorem, the operator $\mathcal{P}: \mathcal{C} \rightarrow \mathcal{C}$ is completely continuous.

Finally, we show that there exists an open set $U \subset \mathcal{C}$ with $x \neq \theta \mathcal{P} x$ for $\theta \in(0,1)$ and $x \in \partial U$. Let $x$ be a solution. Then, for $t \in[0, T]$, and following the similar computations as in the first step, we have

$$
|x(t)| \leq \Phi(\|x\|)\left\{J^{q} p(s)(T)+\frac{|\alpha|}{|\Lambda|}\left(\left|v_{4}\right|+T\left|v_{3}\right|\right) I_{\eta}^{\gamma, \delta} J^{q} p(s)(\zeta)\right.
$$




$$
\left.+\frac{1}{|\Lambda|}\left(\left|v_{2}\right|+T\left|v_{1}\right|\right)\left(|\beta|^{\rho} I^{p} J^{q} p(s)(\xi)+J^{q} p(s)(T)\right)\right\}
$$

which leads to

$$
\|x\| / \Phi(\|x\|) \leq J^{q} p(s)(T)+A_{1}+A_{2} .
$$

In view of $\left(H_{4}\right)$, there exists $N$ such that $\|x\| \neq N$. Let us set

$$
U=\{x \in \mathcal{C}:\|x\|<N\}
$$

We see that the operator $\mathcal{P}: \bar{U} \rightarrow C([0, T], \mathbb{R})$ is continuous and completely continuous. From the choice of $U$, there is no $x \in \partial U$ such that $x=\theta \mathcal{P} x$ for some $\theta \in(0,1)$. Consequently, by the nonlinear alternative of Leray-Schauder type, we deduce that $\mathcal{P}$ has a fixed point $x \in \bar{U}$ which is a solution of the boundary value problem (1.1). This completes the proof.

\subsection{Existence and uniqueness result via Banach's fixed point theo- rem}

Theorem 3. Let $f:[0, T] \times \mathbb{R} \rightarrow \mathbb{R}$ be a continuous function satisfying the assumptions $\left(H_{1}\right)$. Then there exists a unique solution for problem (1.1) on $[0, T]$ provided that $L \Psi<1$, where $\Psi$ is defined by (3.2).

Proof. Using the operator $\mathcal{P}$ defined by (3.1), we transform problem (1.1) into a fixed point problem as $x=\mathcal{P} x$. Observe that the fixed points of the operator $\mathcal{P}$ are solutions of problem (1.1). Applying the Banach contraction mapping principle, we shall show that $\mathcal{P}$ has a unique fixed point.

Letting $\sup _{t \in[0, T]}|f(t, 0)|=M<\infty$ and $r \geq M \Psi /(1-L \Psi)$, we define a closed ball $B_{r}=\{x \in \mathcal{C}:\|x\| \leq r\}$ and show that $\mathcal{P} B_{r} \subset B_{r}$. Then, using $|f(s, x(s))| \leq|f(s, x(s))-f(s, 0)|+|f(s, 0)| \leq L\|x\|+M \leq L r+M$, for any $x \in B_{r}$, we have

$$
\begin{aligned}
|(\mathcal{P} x)(t)| \leq \sup _{t \in[0, T]}\left\{J^{q}|f(s, x(s))|(t)+\frac{|\alpha|}{|\Lambda|}\left(\left|v_{4}\right|+T\left|v_{3}\right|\right) I_{\eta}^{\gamma, \delta} J^{q}|f(s, x(s))|(\zeta)\right. \\
\left.\quad+\frac{1}{|\Lambda|}\left(\left|v_{2}\right|+T\left|v_{1}\right|\right)\left(|\beta|^{\rho} I^{p} J^{q}|f(s, x(s))|(\xi)+J^{q}|f(s, x(s))|(T)\right)\right\} \\
\leq(L\|x\|+M) J^{q}(1)(T)+(L\|x\|+M) \frac{|\alpha|}{|\Lambda|}\left(\left|v_{4}\right|+T\left|v_{3}\right|\right) I_{\eta}^{\gamma, \delta} J^{q}(1)(\zeta) \\
\quad+(L\|x\|+M) \frac{1}{|\Lambda|}\left(\left|v_{2}\right|+T\left|v_{1}\right|\right)\left(|\beta|{ }^{\rho} I^{p} J^{q}(1)(\xi)+J^{q}(1)(T)\right) \\
\leq(L r+M)\left\{\frac{T^{q}}{\Gamma(q+1)}+\frac{|\alpha|\left(\left|v_{4}\right|+T\left|v_{3}\right|\right)}{|\Lambda|} \frac{\zeta^{q} \Gamma(\gamma+(q / \eta)+1)}{\Gamma(q+1) \Gamma(\gamma+(q / \eta)+\delta+1)}\right. \\
\left.\quad+\frac{\left(\left|v_{2}\right|+T\left|v_{1}\right|\right)}{|\Lambda|}\left(\frac{1}{\Gamma(q+1)} \frac{\xi^{q+\rho p}}{\rho^{p}} \frac{\Gamma(q+\rho / \rho)}{\Gamma(q+\rho p+\rho / \rho)}+\frac{T^{q}}{\Gamma(q+1)}\right)\right\} \\
\leq(L r+M) \Psi \leq r, \quad
\end{aligned}
$$

which implies that $\mathcal{P} B_{r} \subset B_{r}$. 
Next, we let $x, y \in \mathcal{C}$. Then for $t \in[0, T]$, we have

$$
\begin{aligned}
\mid \mathcal{P} x( & t)-\mathcal{P} y(t) \mid \leq \sup _{t \in[0, T]}\left\{J^{q}|f(s, x(s))-f(s, y(s))|(t)\right. \\
& +\frac{|\alpha|}{|\Lambda|}\left(\left|v_{4}\right|+T\left|v_{3}\right|\right) I_{\eta}^{\gamma, \delta} J^{q}|f(s, x(s))-f(s, y(s))|(\zeta) \\
& +\frac{1}{|\Lambda|}\left(\left|v_{2}\right|+T\left|v_{1}\right|\right)\left(\beta^{\rho} I^{p} J^{q}|f(s, x(s))-f(s, y(s))|(\xi)\right. \\
& \left.\left.+J^{q}|f(s, x(s))-f(s, y(s))|(T)\right)\right\} \\
\leq & L\|x-y\| J^{q}(1)(T)+L\|x-y\| \frac{|\alpha|}{|\Lambda|}\left(\left|v_{4}\right|+T\left|v_{3}\right|\right) I_{\eta}^{\gamma, \delta} J^{q}(1)(\zeta) \\
& +L\|x-y\| \frac{1}{|\Lambda|}\left(\left|v_{2}\right|+T\left|v_{1}\right|\right)\left(|\beta|^{\rho} I^{p} J^{q}(1)(\xi)+J^{q}(1)(T)\right) \\
= & L \Psi\|x-y\|,
\end{aligned}
$$

which leads to $\|\mathcal{P} x-\mathcal{P} y\| \leq L \Psi\|x-y\|$. As $L \Psi<1, \mathcal{P}$ is a contraction. Therefore, we deduce by Banach's contraction mapping principle that $\mathcal{P}$ has a fixed point which corresponds to the unique solution of problem (1.1). The proof is completed.

\subsection{Existence and uniqueness result via nonlinear contractions}

Definition 6. Let $E$ be a Banach space and let $\mathcal{F}: E \rightarrow E$ be a mapping. $\mathcal{F}$ is said to be a nonlinear contraction if there exists a continuous nondecreasing function $\Theta: \mathbb{R}^{+} \rightarrow \mathbb{R}^{+}$such that $\Theta(0)=0$ and $\Theta(\varepsilon)<\varepsilon$ for all $\varepsilon>0$ with the property:

$$
\|\mathcal{F} x-\mathcal{F} y\| \leq \Theta(\|x-y\|), \quad \forall x, y \in E .
$$

Lemma 6. (Boyd and Wong) [8]. Let $E$ be a Banach space and let $\mathcal{F}: E \rightarrow E$ be a nonlinear contraction. Then $\mathcal{F}$ has a unique fixed point in $E$.

Theorem 4. Let $f:[0, T] \times \mathbb{R} \rightarrow \mathbb{R}$ be a continuous function satisfying the assumption:

$$
\left(H_{5}\right)|f(t, x)-f(t, y)| \leq z(t) \frac{|x-y|}{A^{*}+|x-y|}, \text { for } t \in[0, T], x, y \geq 0,
$$

where $z:[0, T] \rightarrow \mathbb{R}^{+}$is continuous and

$$
\begin{aligned}
A^{*}:= & J^{q} z(T)+\frac{1}{|\Lambda|}\left[|\alpha|\left(\left|v_{4}\right|+T\left|v_{3}\right|\right) I_{\eta}^{\gamma, \delta} J^{q} z(\zeta)\right. \\
& \left.+\left(\left|v_{2}\right|+T\left|v_{1}\right|\right)\left(|\beta|^{\rho} I^{p} J^{q} z(\xi)+J^{q} z(T)\right)\right] .
\end{aligned}
$$

Then problem (1.1) has a unique solution on $[0, T]$.

Proof. Let us introduce a continuous nondecreasing function $\Theta: \mathbb{R}^{+} \rightarrow \mathbb{R}^{+}$ as follows

$$
\Theta(\varepsilon)=A^{*} \varepsilon /\left(A^{*}+\varepsilon\right), \quad \forall \varepsilon \geq 0
$$


Note that the function $\Theta$ satisfies $\Theta(0)=0$ and $\Theta(\varepsilon)<\varepsilon$ for all $\varepsilon>0$.

For any $x, y \in \mathcal{C}$ and for each $t \in[0, T]$, we have

$$
\begin{aligned}
\mid \mathcal{P} x( & t)-\mathcal{P} y(t) \mid \leq \sup _{t \in[0, T]}\left\{J^{q}|f(s, x(s))-f(s, y(s))|(t)\right. \\
& +\frac{|\alpha|}{|\Lambda|}\left(\left|v_{4}\right|+T\left|v_{3}\right|\right) I_{\eta}^{\gamma, \delta} J^{q}|f(s, x(s))-f(s, y(s))|(\zeta) \\
& +\frac{1}{|\Lambda|}\left(\left|v_{2}\right|+T\left|v_{1}\right|\right)\left(|\beta|^{\rho} I^{p} J^{q}|f(s, x(s))-f(s, y(s))|(\xi)\right. \\
& \left.\left.+J^{q}|f(s, x(s))-f(s, y(s))|(T)\right)\right\} \\
\leq & J^{q}\left(z(s) \frac{|x-y|}{A^{*}+|x-y|}\right)(T)+\frac{|\alpha|}{|\Lambda|}\left(\left|v_{4}\right|+T\left|v_{3}\right|\right) I_{\eta}^{\gamma, \delta} \\
& \times J^{q}\left(z(s) \frac{|x-y|}{A^{*}+|x-y|}\right)(\zeta)+\frac{1}{|\Lambda|}\left(\left|v_{2}\right|+T\left|v_{1}\right|\right. \\
& \times\left\{|\beta|^{\rho} I^{p} J^{q}\left(z(s) \frac{|x-y|}{A^{*}+|x-y|}\right)(\xi)+J^{q}\left(z(s) \frac{|x-y|}{A^{*}+|x-y|}\right)(T)\right\} \\
\leq & \frac{\Theta(\|x-y\|)}{A^{*}}\left[J^{q} z(T)+\frac{|\alpha|}{|\Lambda|}\left(\left|v_{4}\right|+T\left|v_{3}\right|\right) I_{\eta}^{\gamma, \delta} J^{q} z(\zeta)\right. \\
& \left.+\frac{1}{|\Lambda|}\left(\left|v_{2}\right|+T\left|v_{1}\right|\right)\left\{|\beta|{ }^{\rho} I^{p} J^{q} z(\xi)+J^{q} z(T)\right\}\right]=\Theta(\|x-y\|) .
\end{aligned}
$$

This implies that $\|\mathcal{P} x-\mathcal{P} y\| \leq \Theta(\|x-y\|)$. Therefore $\mathcal{P}$ is a nonlinear contraction. Hence, by Lemma 6 the operator $\mathcal{P}$ has a unique fixed point which is the unique solution of problem (1.1). This completes the proof.

\subsection{Existence result via Schaefer fixed point theorem}

Lemma 7. [28] Let $X$ be a Banach space. Assume that $T: X \rightarrow X$ is a completely continuous operator and the set $V=\{u \in X \mid u=\mu T u, 0<\mu<1\}$ is bounded. Then $T$ has a fixed point in $X$.

Theorem 5. Let $f:[0, T] \times \mathbb{R} \rightarrow \mathbb{R}$ be a continuous function. Assume that there exists a positive constant $L_{1}$ such that $|f(t, x)| \leq L_{1}$ for $t \in[0, T], x \in \mathbb{R}$. Then the boundary value problem (1.1) has at least one solution on $[0, T]$.

Proof. In the first step, we show that the operator $\mathcal{P}$ defined by (3.1) is completely continuous. Observe that continuity of $f$ implies continuity of $\mathcal{P}$. For a positive constant $r$, let $B_{r}=\{x \in \mathcal{C}:\|x\| \leq r\}$ be a bounded set in $\mathcal{C}$. Then, for $t \in[0, T]$, we obtain

$$
\begin{aligned}
& |\mathcal{P} x(t)| \leq J^{q}|f(s, x(s))|(t)+\frac{|\alpha|}{|\Lambda|}\left(\left|v_{4}\right|+T\left|v_{3}\right|\right) I_{\eta}^{\gamma, \delta} J^{q}|f(s, x(s))|(\zeta) \\
& \quad+\frac{1}{|\Lambda|}\left(\left|v_{2}\right|+T\left|v_{1}\right|\right)\left(|\beta|{ }^{\rho} I^{p} J^{q}|f(s, x(s))|(\xi)+J^{q}|f(s, x(s))|(T)\right) \\
& \leq L_{1} J^{q}(1)(T)+L_{1} \frac{|\alpha|}{|\Lambda|}\left(\left|v_{4}\right|+T\left|v_{3}\right|\right) I_{\eta}^{\gamma, \delta} J^{q}(1)(\zeta)
\end{aligned}
$$




$$
\begin{aligned}
& +L_{1} \frac{1}{|\Lambda|}\left(\left|v_{2}\right|+T\left|v_{1}\right|\right)\left(|\beta|^{\rho} I^{p} J^{q}(1)(\xi)+J^{q}(1)(T)\right) \\
\leq & L_{1}\left\{\frac{T^{q}}{\Gamma(q+1)}+\frac{|\alpha|\left(\left|v_{4}\right|+T\left|v_{3}\right|\right)}{|\Lambda|} \frac{\zeta^{q} \Gamma(\gamma+(q / \eta)+1)}{\Gamma(q+1) \Gamma(\gamma+(q / \eta)+\delta+1)}\right. \\
& \left.+\frac{\left(\left|v_{2}\right|+T\left|v_{1}\right|\right)}{|\Lambda|}\left(\frac{1}{\Gamma(q+1)} \frac{\xi^{q+\rho p}}{\rho^{p}} \frac{\Gamma((q+\rho) / \rho)}{\Gamma((q+\rho p+\rho \rho) /)}+\frac{T^{q}}{\Gamma(q+1)}\right)\right\},
\end{aligned}
$$

which, by virtue of (3.2), yields

$$
\|\mathcal{P} x\| \leq L_{1} \Psi .
$$

Next we show that the operator $\mathcal{P}$ maps bounded sets into equicontinuous sets of $\mathcal{C}$. Let $\tau_{1}, \tau_{2} \in[0, T]$ with $\tau_{1}<\tau_{2}$ and $x \in B_{r}$. Then we have

$$
\begin{aligned}
\mid \mathcal{P} x & \left(\tau_{2}\right)-\mathcal{P} x\left(\tau_{1}\right)|\leq| J^{q} f(s, x(s))\left(\tau_{2}\right)-J^{q} f(s, x(s))\left(\tau_{1}\right) \mid \\
& +\frac{|\alpha|\left|v_{2}\right|\left|\tau_{2}-\tau_{1}\right|}{|\Lambda|} I_{\eta}^{\gamma, \delta} J^{q}|f(s, x(s))|(\zeta) \\
& +\frac{\left|v_{1}\right|\left|\tau_{2}-\tau_{1}\right|}{|\Lambda|}\left(|\beta|^{\rho} I^{p} J^{q}|f(s, x(s))|(\xi)+J^{q}|f(s, x(s))|(T)\right) \\
\leq & \frac{L_{1}}{\Gamma(q)}\left|\int_{0}^{\tau_{1}}\left[\left(\tau_{2}-s\right)^{q-1}-\left(\tau_{1}-s\right)^{q-1}\right] d s+\int_{\tau_{1}}^{\tau_{2}}\left(\tau_{2}-s\right)^{q-1} d s\right| \\
& +\frac{L_{1}|\alpha|\left|v_{2}\right|\left|\tau_{2}-\tau_{1}\right|}{|\Lambda|} I_{\eta}^{\gamma, \delta} J^{q}(\zeta)+\frac{L_{1}\left|v_{1}\right|\left|\tau_{2}-\tau_{1}\right|}{|\Lambda|}\left(|\beta|{ }^{\rho} I^{p} J^{q}(\xi)+J^{q}(T)\right) \\
\leq & \frac{L_{1}}{\Gamma(q+1)}\left(\tau_{2}^{q}-\tau_{1}^{q}\right)+\frac{L_{1}|\alpha|\left|v_{2}\right|\left|\tau_{2}-\tau_{1}\right|}{|\Lambda|} \frac{\zeta^{q} \Gamma(\gamma+(q / \eta)+1)}{\Gamma(q+1) \Gamma(\gamma+(q / \eta)+\delta+1)} \\
& +\frac{L_{1}\left|v_{1}\right|\left|\tau_{2}-\tau_{1}\right|}{|\Lambda|}\left(\frac{1}{\Gamma(q+1)} \frac{\xi^{q+\rho p}}{\rho^{p}} \frac{\Gamma((q+\rho) / \rho)}{\Gamma((q+\rho p+\rho) / \rho)}+\frac{T^{q}}{\Gamma(q+1)}\right) .
\end{aligned}
$$

As $\tau_{2}-\tau_{1} \rightarrow 0$, the right-hand side of the above inequality tends to zero independently of $x \in B_{r}$. Therefore by the Arzelá-Ascoli theorem the operator $\mathcal{P}: \mathcal{C} \rightarrow \mathcal{C}$ is completely continuous.

Next, we consider the set $V=\{x \in \mathcal{C}: x=\mu \mathcal{P} x, 0<\mu<1\}$. In order to show that $V$ is bounded, let $x \in V$ and $t \in[0, T]$. Then

$$
\begin{aligned}
\|x\| \leq & L_{1}\left\{\frac{T^{q}}{\Gamma(q+1)}+\frac{|\alpha|\left(\left|v_{4}\right|+T\left|v_{3}\right|\right)}{|\Lambda|} \frac{\zeta^{q} \Gamma(\gamma+(q / \eta)+1)}{\Gamma(q+1) \Gamma(\gamma+(q / \eta)+\delta+1)}\right. \\
& \left.+\frac{\left(\left|v_{2}\right|+T\left|v_{1}\right|\right)}{|\Lambda|}\left(\frac{1}{\Gamma(q+1)} \frac{\xi^{q+\rho p}}{\rho^{p}} \frac{\Gamma\left(\frac{q+\rho}{\rho}\right)}{\Gamma\left(\frac{q+\rho p+\rho}{\rho}\right)}+\frac{T^{q}}{\Gamma(q+1)}\right)\right\}=M_{1} .
\end{aligned}
$$

Therefore, $\mathrm{V}$ is bounded. Hence, by Lemma 7, problem (1.1) has at least one solution on $[0, T]$.

\subsection{Existence result via Leray-Schauder's Degree Theory}

Theorem 6. Let $f:[0, T] \times \mathbb{R} \rightarrow \mathbb{R}$ be a continuous function. Suppose that 
$\left(H_{6}\right)$ there exist constants $0 \leq \nu<\Psi^{-1}$, and $M>0$ such that

$$
|f(t, x)| \leq \nu|x|+M \text { for all }(t, x) \in[0, T] \times \mathbb{R},
$$

where $\Psi$ is defined by (3.2).

Then the boundary value problem (1.1) has at least one solution on $[0, T]$.

Proof. Let us consider the operator equation

$$
x=\mathcal{P} x,
$$

where the operator $\mathcal{P}: \mathcal{C} \rightarrow \mathcal{C}$ is defined by (3.1). Our proof will be complete once we establish the existence of at least one solution $x \in \mathcal{C}$ for (3.4). Set a ball $B_{R} \subset C[0, T]$ with a constant radius $R>0$ as follows

$$
B_{R}=\left\{x \in \mathcal{C}: \max _{t \in[0, T]}|x(t)|<R\right\}
$$

and show that the operator $\mathcal{P}: \bar{B}_{R} \rightarrow C[0, T]$ satisfies the condition

$$
x \neq \theta \mathcal{P} x, \quad \forall x \in \partial B_{R}, \quad \forall \theta \in[0,1] .
$$

Define $H(\theta, x)=\theta \mathcal{P} x, x \in \mathcal{C}, \theta \in[0,1]$. We know from Theorem 2 that the operator $\mathcal{P}$ is continuous, uniformly bounded and equicontinuous. Then, by the Arzelá-Ascoli theorem, a continuous map $h_{\theta}$ defined by $h_{\theta}(x)=x-H(\theta, x)=$ $x-\theta \mathcal{P} x$ is completely continuous. If (3.5) holds, then the following LeraySchauder degrees are well defined and by the homotopy invariance of topological degree, it follows that

$$
\begin{aligned}
\operatorname{deg}\left(h_{\theta}, B_{R}, 0\right) & =\operatorname{deg}\left(I-\theta \mathcal{P}, B_{R}, 0\right)=\operatorname{deg}\left(h_{1}, B_{R}, 0\right) \\
& =\operatorname{deg}\left(h_{0}, B_{R}, 0\right)=\operatorname{deg}\left(I, B_{R}, 0\right)=1 \neq 0, \quad 0 \in B_{R},
\end{aligned}
$$

where $I$ denotes the unit operator. By the nonzero property of Leray-Schauder degree, we have $h_{1}(x)=x-\mathcal{P} x=0$ for at least one $x \in B_{R}$. Let us assume that $x=\theta \mathcal{P} x$ for some $\theta \in[0,1]$ and for all $t \in[0, T]$ so that

$$
\begin{aligned}
|x(t)|= & |\theta \mathcal{P} x(t)| \\
\leq & J^{q}|f(s, x(s))|(t)+\frac{|\alpha|}{|\Lambda|}\left(\left|v_{4}\right|+T\left|v_{3}\right|\right) I_{\eta}^{\gamma, \delta} J^{q}|f(s, x(s))|(\zeta) \\
& +\frac{1}{|\Lambda|}\left(\left|v_{2}\right|+T\left|v_{1}\right|\right)\left(|\beta|{ }^{\rho} I^{p} J^{q}|f(s, x(s))|(\xi)+J^{q}|f(s, x(s))|(T)\right) \\
\leq & (\nu|x|+M) J^{q} p(s)(T)+(\nu|x|+M) \frac{|\alpha|}{|\Lambda|}\left(\left|v_{4}\right|+T\left|v_{3}\right|\right) I_{\eta}^{\gamma, \delta} J^{q} p(s)(\zeta) \\
& +(\nu|x|+M) \frac{1}{|\Lambda|}\left(\left|v_{2}\right|+T\left|v_{1}\right|\right)\left(|\beta|^{\rho} I^{p} J^{q} p(s)(\xi)+J^{q} p(s)(T)\right) \\
= & (\nu|x|+M) \Psi,
\end{aligned}
$$

which, on taking the norm $\sup _{t \in[0, T]}|x(t)|=\|x\|$ and solving for $\|x\|$, yields

$$
\|x\| \leq \frac{M \Psi}{1-\nu \Psi}
$$

If $R=\frac{M \Psi}{1-\nu \Psi}+1$, the inequality (3.5) holds. This completes the proof. 


\section{Examples}

In this section, we illustrate the results obtained in the last section.

Example 1. Consider the following fractional-order boundary value problem involving nonlocal Erdélyi-Kober and generalized Riemann-Liouville fractional integral conditions

$$
\left\{\begin{array}{l}
{ }^{c} D^{\frac{5}{4}} x(t)=\frac{1}{15} \frac{e^{-2 t}}{(t+3)^{2}} \frac{|x(t)|}{1+3|x(t)|}+\frac{1}{4} \log (t+1), \quad t \in[0,5 / 2], \\
x(0)=\frac{2}{\sqrt{3}} I_{\frac{2}{3}}^{\frac{\sqrt{\pi}}{4}}, \frac{2}{e^{2}} x\left(\frac{1}{2}\right), \quad x\left(\frac{5}{2}\right)=\frac{3}{14} \frac{5}{13} I^{\frac{1}{\sqrt{2}}} x\left(\frac{3}{2}\right) .
\end{array}\right.
$$

Here $q=5 / 4, T=5 / 2, \alpha=2 / \sqrt{3}, \gamma=\sqrt{\pi} / 4, \delta=2 / e^{2}, \eta=2 / 3, \zeta=1 / 2, \beta=$ $3 / 14, \rho=5 / 13, p=1 / \sqrt{2}, \xi=3 / 2$ and $f(t, x)=(1 / 15)\left(e^{-2 t} /(t+3)^{2}\right)(|x| /(1+$ $3|x|))+(1 / 4) \log (t+1)$. Computing the given data, we obtain the following constants $v_{1}=-0.122299372, v_{2}=0.4460979363, v_{3}=0.6773267311$, $v_{4}=2.206732908$ and $\Lambda=0.0322720081 \neq 0$. Since $|f(t, x)-f(t, y)| \leq$ $(1 / 135)|x-y|$, the condition $\left(H_{1}\right)$ holds with $L=1 / 135$. In addition, we find that $|f(t, x)| \leq e^{-2 t} /\left(15(t+3)^{2}\right)+(1 / 4) \log (t+1)$ and $\Psi_{1}=0.9688798089<1$. Therefore, by applying Theorem 1, problem (4.1) has at least one solution on $[0,5 / 2]$.

Example 2. Consider the following fractional-order boundary value problem involving nonlocal Erdélyi-Kober and generalized Riemann-Liouville fractional integral conditions

$$
\left\{\begin{array}{l}
{ }^{c} D^{\frac{11}{6}} x(t)=\frac{\left(t^{\frac{1}{3}}+t^{\frac{1}{2}}\right)}{15}\left(\frac{2 x^{2}(t)+3|x(t)|}{1+|x(t)|}+2\right), \quad t \in[0,1 / 2], \\
x(0)=\frac{1}{\sqrt{\pi}} I_{\frac{5}{\pi^{2}}}^{\frac{4}{\sqrt{10}}} x\left(\frac{3}{8}\right), \quad x\left(\frac{1}{2}\right)=\frac{\sqrt{7}}{12} \frac{11}{18} I^{\frac{5}{7}} x\left(\frac{1}{4}\right) .
\end{array}\right.
$$

Here $q=11 / 6, T=1 / 2, \alpha=1 / \sqrt{\pi}, \gamma=4 / 7, \delta=3 / \sqrt{10}, \eta=5 / \pi^{2}, \zeta=1 / 8$, $\beta=\sqrt{7} / 12, \rho=11 / 18, p=5 / 7, \xi=1 / 4$ and $f(t, x)=\left(\left(t^{1 / 3}+t^{1 / 2}\right) / 15\right)\left(\left(\left(2 x^{2}+\right.\right.\right.$ $3|x|) /(1+|x|))+2)$. By direct computation of given constants, we have $v_{1}=$ $0.6273547690, v_{2}=0.02136672311, v_{3}=0.8527688407, v_{4}=0.4777750041$ and $\Lambda=0.3179553030 \neq 0$. Choosing $p(t)=\left(t^{\frac{1}{3}}+t^{\frac{1}{2}}\right) / 15$ and $\Phi(|x|)=2|x|+5$, we have $A_{1}=0.0001321467571$ and $A_{2}=0.01053345765$. Then there exists a positive constant $N>0.1070970081$ satisfying inequality in $\left(H_{4}\right)$. Therefore, all conditions of Theorem 2 are fulfilled. Hence, problem (4.2) has at least one solution on $[0,1 / 2]$.

Example 3. Consider the following fractional-order boundary value problem involving nonlocal Erdélyi-Kober and generalized Riemann-Liouville fractional integral conditions

$$
\left\{\begin{array}{l}
{ }^{c} D^{\frac{3}{2}} x(t)=\frac{1}{8}\left(\frac{x^{2}(t)+4|x(t)|}{3+|x(t)|}\right) \cos ^{2} t+\frac{1}{2 \sin ^{2} t}, \quad t \in[0,3 / 2], \\
x(0)=\frac{3}{7} I^{\frac{1}{2}, \frac{2}{9}} \frac{\sqrt{5}}{9} x\left(\frac{1}{2}\right), \quad x\left(\frac{3}{2}\right)=\frac{4}{5} \frac{3}{7} I^{\frac{6}{11}} x\left(\frac{11}{8}\right) .
\end{array}\right.
$$


Here $q=3 / 2, T=3 / 2, \alpha=3 / 7, \gamma=1 / 2, \delta=2 / \sqrt{3}, \eta=\sqrt{5} / 9, \zeta=1 / 2$, $\beta=4 / 5, \rho=3 / 7, p=6 / 11, \xi=11 / 8$ and $f(t, x)=(1 / 8)\left(\left(x^{2}+4|x|\right) /(3+\right.$ $|x|)) \cos ^{2} t+(1 / 2) \sin ^{2} t$. Using the given data, we find that $v_{1}=0.7451980583$, $v_{2}=0.02931419848, v_{3}=-0.910393214, v_{4}=0.487833885, \Lambda=0.3368454165$ and $\Psi=5.736302996$. Since $|f(t, x)-f(t, y)| \leq(1 / 6)|x-y|$, the condition $\left(H_{1}\right)$ is satisfied with $L=1 / 6$. Obviously $L \Psi=0.9560504993<1$. Thus, by Theorem 3, problem (4.3) has a unique solution on $[0,3 / 2]$.

Example 4. Consider the following fractional-order boundary value problem

$$
\left\{\begin{array}{l}
{ }^{c} D^{\frac{7}{5}} x(t)=\frac{1}{3(t+2)^{3}}\left(\sin |x(t)|+\frac{|x(t)|}{1+|x(t)|}\right)+\frac{1}{2}\left(t^{\frac{1}{4}}+1\right), t \in[0,2], \\
x(0)=\frac{\sqrt{2}}{5} I_{\frac{3}{7}}^{\frac{\pi}{2}}, \frac{1}{4} x\left(\frac{1}{2}\right), \quad x(2)=\frac{\sqrt{3}}{4} \frac{8}{15} I^{\frac{13}{19}} x\left(\frac{3}{2}\right),
\end{array}\right.
$$

where $q=7 / 5, T=2, \alpha=\sqrt{2} / 5, \gamma=\pi / 2, \delta=1 / 4, \eta=3 / 7, \zeta=1 / 2$, $\beta=\sqrt{3} / 4, \rho=8 / 15, p=13 / 19, \xi=3 / 2$ and $f(t, x)=\left(1 /\left(3(t+2)^{3}\right)\right)(\sin |x|+$ $(|x|) /(1+|x|))+(1 / 2)\left(t^{\frac{1}{4}}+1\right)$. Using the given values, we find that $v_{1}=$ $0.7681027408, v_{2}=0.09689608493, v_{3}=0.2722751129, v_{4}=1.416898935$, $\Lambda=1.114706347$. Choosing $z(t)=t^{\frac{1}{3}} / 12$, we get $A^{*}=0.4296509478$. Clearly the condition $\left(H_{2}\right)$ holds true as

$$
|f(t, x)-f(t, y)| \leq \frac{t^{\frac{1}{3}}}{12}\left(\frac{|x-y|}{0.4296509478+|x-y|}\right)
$$

Therefore, from Theorem 4, problem (4.4) has a unique solution on $[0,2]$.

Example 5. Consider the following problem of Caputo fractional differential equation supplemented with nonlocal Erdélyi-Kober and generalized RiemannLiouville fractional integral conditions

$$
\left\{\begin{array}{l}
{ }^{c} D^{\frac{27}{17}} x(t)=\frac{1}{2} \tan ^{-1}\left(\frac{x^{2}(t)+|x(t)|}{1+|x(t)|}\right)\left(3 \cos ^{2} t+1\right)+\pi, t \in\left[0, \frac{5}{2}\right], \\
x(0)=\frac{1}{\sqrt{7}} I_{\frac{5}{9}}^{\frac{5}{8}, \frac{7}{13}} x(1), \quad x\left(\frac{5}{2}\right)=\frac{1}{\sqrt{3}}{ }^{\frac{4}{3}} I^{\frac{\sqrt{7}}{6}} x(2),
\end{array}\right.
$$

where $q=27 / 17, T=5 / 2, \alpha=1 / \sqrt{7}, \gamma=5 / 8, \delta=7 / 13, \eta=5 / 9, \zeta=1$, $\beta=1 / \sqrt{3}, \rho=4 / 3, p=\sqrt{7} / 6, \xi=2$ and $f(t, x)=(1 / 2) \tan ^{-1}\left(\left(x^{2}+|x|\right) /(1+\right.$ $|x|))\left(3 \cos ^{2} t+1\right)+\pi$. By the given data, we find that $v_{1}=0.6863802809, v_{2}=$ $0.2019299623, v_{3}=0.1702153301, v_{4}=1.218454775, \Lambda=0.8706949059 \neq 0$. It is clear that $|f(t, x)| \leq 2 \pi$. Thus all the assumptions of Theorem 5 are satisfied. In consequence, problem (4.5) has at least one solution on $[0,5 / 2]$.

Example 6. Consider the following nonlocal nonlinear fractional-order problem involving Erdélyi-Kober and generalized Riemann-Liouville fractional integral 
conditions

$$
\left\{\begin{array}{l}
{ }^{c} D^{\frac{43}{26}} x(t)=\frac{\left(\sin ^{2} t+1\right)}{120}\left(\frac{x^{2}(t)}{1+|x(t)|}\right) e^{-t}+\frac{2|x(t)|}{1+3|x(t)|}+\frac{1}{3}, t \in[0,3], \\
x(0)=\frac{3}{16} I_{\frac{1}{\sqrt{6}}}^{\frac{4}{\sqrt{5}}}, \frac{\sqrt{3}}{2} x\left(\frac{3}{2}\right), \quad x(3)=\frac{2}{\sqrt{5}}{ }^{\frac{\sqrt{e}}{2}} I^{\frac{3}{7}} x\left(\frac{5}{2}\right) .
\end{array}\right.
$$

Here $q=43 / 26, T=3, \alpha=3 / 16, \gamma=4 / \sqrt{5}, \delta=\sqrt{3} / 2, \eta=1 / \sqrt{6}, \zeta=3 / 2$, $\beta=2 / \sqrt{5}, \rho=\sqrt{e} / 2, p=3 / 7, \xi=5 / 2$ and $f(t, x)=\left(\left(\sin ^{2} t+1\right) / 120\right)\left(x^{2} /(1+\right.$ $|x|)) e^{-t}+((2|x|) /(1+3|x|))+(1 / 3)$. With the given values, we have that $v_{1}=$ $0.9213181351, v_{2}=0.06775588797, v_{3}=-0.658410315, v_{4}=0.474997666$, $\Lambda=0.3930127883 \neq 0$ and $\Psi=55.66868159$. Also $|f(t, x)| \leq(1 / 60)|x|+1$ with $\nu=(1 / 60)=0.01666666667<0.01796342165=\Psi^{-1}$ and $M=1$. Therefore, the conclusion of Theorem 6 implies that problem (4.6) has at least one solution on $[0,3]$.

\section{References}

[1] P. Agarwal, J. Choi and R.B. Paris. Extended Riemann-Liouville fractional derivative operator and its applications. J. Nonlinear Sci. Appl., 8:451-466, 2015 .

[2] B. Ahmad, A. Alsaedi and B.S. Alghamdi. Analytic approximation of solutions of the forced duffing equation with integral boundary conditions. Nonlinear Anal. Real World Appl., 9(4):1727-1740, 2008. https://doi.org/10.1016/j.nonrwa.2007.05.005.

[3] B. Ahmad and J. Nieto. Riemann-Liouville fractional integro-differential equations with fractional nonlocal integral boundary conditions. Bound. Value Probl., 2011:36, 2011. https://doi.org/10.1186/1687-2770-2011-36.

[4] B. Ahmad and S.K. Ntouyas. Nonlocal fractional boundary value problems with slit-strips integral boundary conditions. Fract. Calc. Appl. Anal., 18(1):261-280, 2015. https://doi.org/10.1515/fca-2015-0017.

[5] B. Ahmad, S.K. Ntouyas and A. Alsaedi. A study of nonlinear fractional differential equations of arbitrary order with Riemann-Liouville type multistrip boundary conditions. Math. Probl. Eng., Art. ID 320415:9 pp, 2013.

[6] B Ahmad, S.K. Ntouyas and J. Tariboon. A study of mixed Hadamard and Riemann-Liouville fractional integro-differential inclusions via endpoint theory. Appl. Math. Lett., 52:9-14, 2016. https://doi.org/10.1016/j.aml.2015.08.002.

[7] Z.B. Bai and W. Sun. Existence and multiplicity of positive solutions for singular fractional boundary value problems. Comput. Math. Appl., 63(9):1369-1381, 2012. https://doi.org/10.1016/j.camwa.2011.12.078.

[8] D.W. Boyd and J.S.W. Wong. On nonlinear contractions. Proc. Amer. Math. Soc., 20:458-464, 1969. https://doi.org/10.1090/S0002-9939-1969-0239559-9.

[9] A.G. Butkovskii, S.S. Postnov and E.A. Postnova. Fractional integro-differential calculus and its control-theoretical applications in mathematical fundamentals and the problem of interpretation. Automation and Remote Control, 74(4):543574, 2013. https://doi.org/10.1134/S0005117913040012. 
[10] J. Choi, D. Ritelli and P. Agarwal. Some new inequalities involving generalized Erdélyi-Kober fractional $q$-integral operator. Applied Mathematical Sciences, 9(72):3577-3591, 2015. https://doi.org/10.12988/ams.2015.53190.

[11] A. Erdélyi and H. Kober. Some remarks on hankel transforms. Quart. J. Math., Oxford, Second Ser., 11(1):212-221, 1940. https://doi.org/10.1093/qmath/os11.1.212.

[12] C. Goodrich. Existence and uniqueness of solutions to a fractional difference equation with nonlocal conditions. Comput. Math. Appl., 61(2):191-202, 2011. https://doi.org/10.1016/j.camwa.2010.10.041.

[13] J.R. Graef, J. Henderson and A. Ouahab. Fractional differential inclusions in the almgren sense. Fract. Calc. Appl. Anal., 18:673-686, 2015. https://doi.org/10.1515/fca-2015-0041.

[14] A. Granas and J. Dugundji. Fixed Point Theory. Springer-Verlag, New York, 2003. https://doi.org/10.1007/978-0-387-21593-8.

[15] U.N. Katugampola. New approach to a generalized fractional integral. Appl. Math. Comput., 218(3):860-865, 2011. https://doi.org/10.1016/j.amc.2011.03.062.

[16] A.A. Kilbas, H.M. Srivastava and J.J. Trujillo. Theory and Applications of Fractional Differential Equations. Elsevier Science B.V., N Amsterdam, 2006.

[17] V. Kiryakova. Generalized Fractional Calculus and Applications. Pitman Research Notes in Math. 301, Longman, Harlow - J. Wiley, N. York, 1994.

[18] J. Klafter, S.C. Lim and R. Metzler. Fractional Dynamics in Physics. World Scientific, Singapore, 2011.

[19] H. Kober. On fractional integrals and derivatives. Quart. J. Math. Oxford, 9:193-211, 1940. https://doi.org/10.1093/qmath/os-11.1.193.

[20] M.A. Krasnoselskii. Two remarks on the method of successive approximations. Uspekhi Mat. Nauk, 10:123-127, 1955.

[21] X. Liu, M. Jia and W. Ge. Multiple solutions of a $p$-Laplacian model involving a fractional derivative. Adv. Difference Equ., 2013:126, 2013. https://doi.org/10.1186/1687-1847-2013-126.

[22] Y. Liu. Multiple positive solutions of bvps for singular fractional differential equations with non-caratheodory nonlinearities. Math. Model. Anal., 19(3):395416, 2014. https://doi.org/10.3846/13926292.2014.925984.

[23] A.B. Malinowska, T. Odzijewicz and D.F.M. Torres. Advanced Methods in the Fractional Calculus of Variations. Springer-Verlag, New York, 2015. https://doi.org/10.1007/978-3-319-14756-7.

[24] S.K. Ntouyas, S. Etemad and J. Tariboon. Existence results for multiterm fractional differential inclusions. Adv. Difference Equ., 2015:140, 2015. https://doi.org/10.1186/s13662-015-0481-z.

[25] D. O'Regan and S. Stanek. Fractional boundary value problems with singularities in space variables. Nonlinear Dynam., 71(4):641-652., 2013. https://doi.org/10.1007/s11071-012-0443-x.

[26] J. Sabatier, O.P. Agrawal and J.A.T. Machado. Advances in Fractional Calculus: Theoretical Developments and Applications in Physics and Engineering. Springer, Dordrecht, 2007. https://doi.org/10.1007/978-1-4020-6042-7. 
[27] S.G. Samko, A.A. Kilbas and O.I. Marichev. On fractional integrals and derivatives Fractional Integrals and Derivatives: Theory and Applications. Gordon and Breach, New York, 1993.

[28] D.R. Smart. Fixed Point Theorems. Cambridge University Press, 1980.

[29] I.N. Sneddon. The use in mathematical analysis of Erdélyi-Kober operators and some of their applications. In H. Ammann and V.A. Solonnikov(Eds.), Fractional Calculus and Its Applications, Proc. Internat. Conf. Held in New Haven, Lecture Notes in Math. 457, pp. 37-79. Springer, N. York, 1975.

[30] J. Tariboon, S.K. Ntouyas and A. Singubol. Boundary value problems for fractional differential equations with fractional multi-term integral conditions. $J$. App. Math., Art. ID 806156:10 pp., 2014.

[31] P. Thiramanus, S.K. Ntouyas and J. Tariboon. Existence and uniqueness results for Hadamard-type fractional differential equations with nonlocal fractional integral boundary conditions. Abstr. Appl. Anal., Art. ID 902054:9 pp., 2014.

[32] N. Thongsalee, S.K. Ntouyas and J. Tariboon. Nonlinear Riemann-Liouville fractional differential equations with nonlocal Erdélyi-Kober fractional integral conditions. Frac. Calc. Appl. Anal., 19:480-497, 2016. https://doi.org/10.1515/fca2016-0025.

[33] R. Čiegis and A. Bugajev. Numerical approximation of one model of the bacterial self-organization. Nonlinear Anal. Model. Control, 17(3):253-270, 2012.

[34] X. Zhang, P. Agarwal, Z. Liu and H. Peng. The general solution for impulsive differential equations with Riemann Liouville fractional-order $q \in(1,2)$. Open Math., 13(1):908-930, 2015. https://doi.org/10.1515/math-2015-0073. 